\title{
Commentary \\ Molecular Mechanism of Processive Stepping of Kinesin Motors
}

Ping Xie $(\mathbb{B}$

check for updates

Citation: Xie, P. Molecular Mechanism of Processive Stepping of Kinesin Motors. Symmetry 2021, 13, 1799. https://doi.org/10.3390/ sym13101799

Academic Editors: Ismini

E. Papageorgiou and

Alexandros Zoumpos

Received: 18 August 2021

Accepted: 24 September 2021

Published: 27 September 2021

Publisher's Note: MDPI stays neutral with regard to jurisdictional claims in published maps and institutional affiliations.

Copyright: (C) 2021 by the author. Licensee MDPI, Basel, Switzerland. This article is an open access article distributed under the terms and conditions of the Creative Commons Attribution (CC BY) license (https:/ / creativecommons.org/licenses/by/ $4.0 /)$.
Key Laboratory of Soft Matter Physics, Institute of Physics, Chinese Academy of Sciences, Beijing 100190, China; pxie@aphy.iphy.ac.cn

\begin{abstract}
Kinesin-1 is a motor protein that can step processively on microtubule by hydrolyzing ATP molecules, playing an essential role in intracellular transports. To better understand the mechanochemical coupling of the motor stepping cycle, numerous structural, biochemical, single molecule, theoretical modeling and numerical simulation studies have been undertaken for the kinesin-1 motor. Recently, a novel ultraresolution optical trapping method was employed to study the mechanics of the kinesin-1 motor and new results were supplemented to its stepping dynamics. In this commentary, the new single molecule results are explained well theoretically with one of the models presented in the literature for the mechanochemical coupling of the kinesin- 1 motor. With the model, various prior experimental results for dynamics of different families of $\mathrm{N}$-terminal kinesin motors have also been explained quantitatively.
\end{abstract}

Keywords: molecular motor; kinesin; mechanochemical coupling mechanism

\section{Introduction}

Kinesin- 1 molecular motor is the firstly discovered member of kinesin superfamily that can be classified into 14 subfamilies and an ungrouped subfamily called orphan kinesins $[1,2]$. Kinesin- 1 has been studied thoroughly and can be regarded as the model system to study the mechanism of the mechanochemical coupling of the kinesin superfamily. Kinesin-1 can step processively on microtubules (MTs) by hydrolyzing ATP molecules, which is responsible for cargo transports in cells. To explore the molecular mechanism of the processive stepping of the kinesin-1, plenty of researches on its structure, its interaction with MT, its motor dynamics, etc., have been undertaken. For example, it was shown structurally that kinesin- 1 is composed of two identical N-terminal motor domains (also called heads) connected together by a common coiled-coil stalk via the two flexible neck linkers (NLs) (with each NL having 14 residues) [3]. The dimer walks on MT in an asymmetric hand-over-hand way, with a step size of about $8 \mathrm{~nm}$, the tubulin repeat period of a MT filament $[4,5]$. The load dependencies of the motor's velocity, run length, ratio of forward to backward steps, dissociation rate, etc., have been well determined [6-13]. Accordingly, numerous models have been proposed to explain the available experimental results [14-22] Nevertheless, the detailed mechanism of the mechanochemical coupling of the kinesin- 1 is still undetermined.

Recently, Sudhakar et al. [23] developed germanium nanospheres with diameter of about $70 \mathrm{~nm}$ as optical trapping probes to study the stepping dynamics of truncated rat kinesin-1 motor, which enhances greatly the spatiotemporal resolution that is limited by using traditional micrometer-sized spheres. Puzzlingly, they found that each 8-nm mechanical step consists of two 4-nm substeps, with the dwell time for the second substep being much shorter than that for the first one. Instead of dissociation from MT, the slip of the motor on MT was observed under high backward loads, which occurs always during the dwell period before the second substep. In this commentary, these new single molecule results measured by Sudhakar et al. [23] are explained well by the theoretical analysis based on one of the models presented in the literature for the mechanochemical coupling 
of kinesin-1 motors [24]. As shown before [18,24-30], this model can explain quantitatively diverse experimental results about the load dependency of the stepping dynamics for various families of $\mathrm{N}$-terminal kinesin motors.

\section{The Model}

Firstly, we describe the major elements, on the basis of which the model is established. (1) The kinesin head in empty, ATP and ADP.Pi states has a strong interaction with MT whereas in ADP state has a weak interaction, as shown by experimental data [31-33]. The strong interaction between the kinesin head and tubulin induces large conformational changes of the tubulin whereas the weak interaction has little effect on the conformation of the tubulin, as indicated by structural and all-atom molecular dynamics (AAMD) simulation data $[34,35]$. The head in ADP state has a much smaller affinity (denoted by $E_{\mathrm{w} 1}$ ) for the tubulin having the large conformational changes than the affinity (denoted by $E_{\mathrm{w} 2}$ ) for the tubulin having no large conformational changes, as shown by AAMD simulation data [35]. This, thus, implies that after Pi release from the ADP.Pi-head bound to MT a short time period $t_{\mathrm{r}}$ (of the order of $10 \mu \mathrm{s}$ [25]) is present when the ADP-head has a much smaller affinity $E_{\mathrm{w} 1}$ for the local tubulin and in time $t_{\mathrm{r}}$, with the changed conformation of the local tubulin returning to the normally unchanged one, the affinity of the ADP-head for the local tubulin changes to $E_{\mathrm{w} 2}$ (see Figure 1). (2) In ADP and empty states, the head has an open nucleotide-binding pocket (NBP) and a conformation, with the NL being incapable of docking onto the head, as indicated by structural and experimental results [36-39], and the head having a high binding energy to the partner ADP-head (with the relative position and orientation of the two heads being shown in Figure 2), as shown by AAMD simulation data [40]. In ATP and ADP.Pi states, NBP closing and a large conformational change of the head can take place, with the NL becoming capable of docking onto the head, as indicated by structural and experimental results [36-39], and the binding energy of the head to the partner ADP-head being reduced significantly, as shown by AAMD simulation data [40]. When NL is in the minus-end orientation, the interference of NL prohibits the closing of NBP and thus the large conformational change of the ATP-head. (3) The head with its NL in the plus-end orientation has a much higher rate of ATP hydrolysis and Pi release than the head with its NL not in the plus-end orientation. This can be explained as follows. The NL not in the plus-end orientation has little interaction with the head, while the NL in the plus-end orientation has a large interaction with the head, enhancing significantly the rate of ATP hydrolysis and Pi release. This is in line with the biochemical data showing that the head with mutation or deletion of its NL has a much smaller ATPase rate than the wild-type case while the mutation or deletion has no influence on ADP-release rate [41], because after ATP binding the NL docks rapidly in the plus-end orientation.

In the main text, we consider the case of saturating ATP and backward load on the stalk of the kinesin dimer (the case of non-saturating ATP is considered in Supplementary Materials). As at saturating ATP after ADP release from a head ATP can bind instantly, the nucleotide-free state of the head is not required to consider. As the head in both ATP and ADP.Pi states has the high binding energy to MT and has the similar conformation, for simplicity, we consider ATP hydrolysis and Pi release as one step of ATP transition to ADP. The pathway for the mechanochemical coupling of the kinesin dimer is schematically shown in Figure 3 [24]. 
(a)
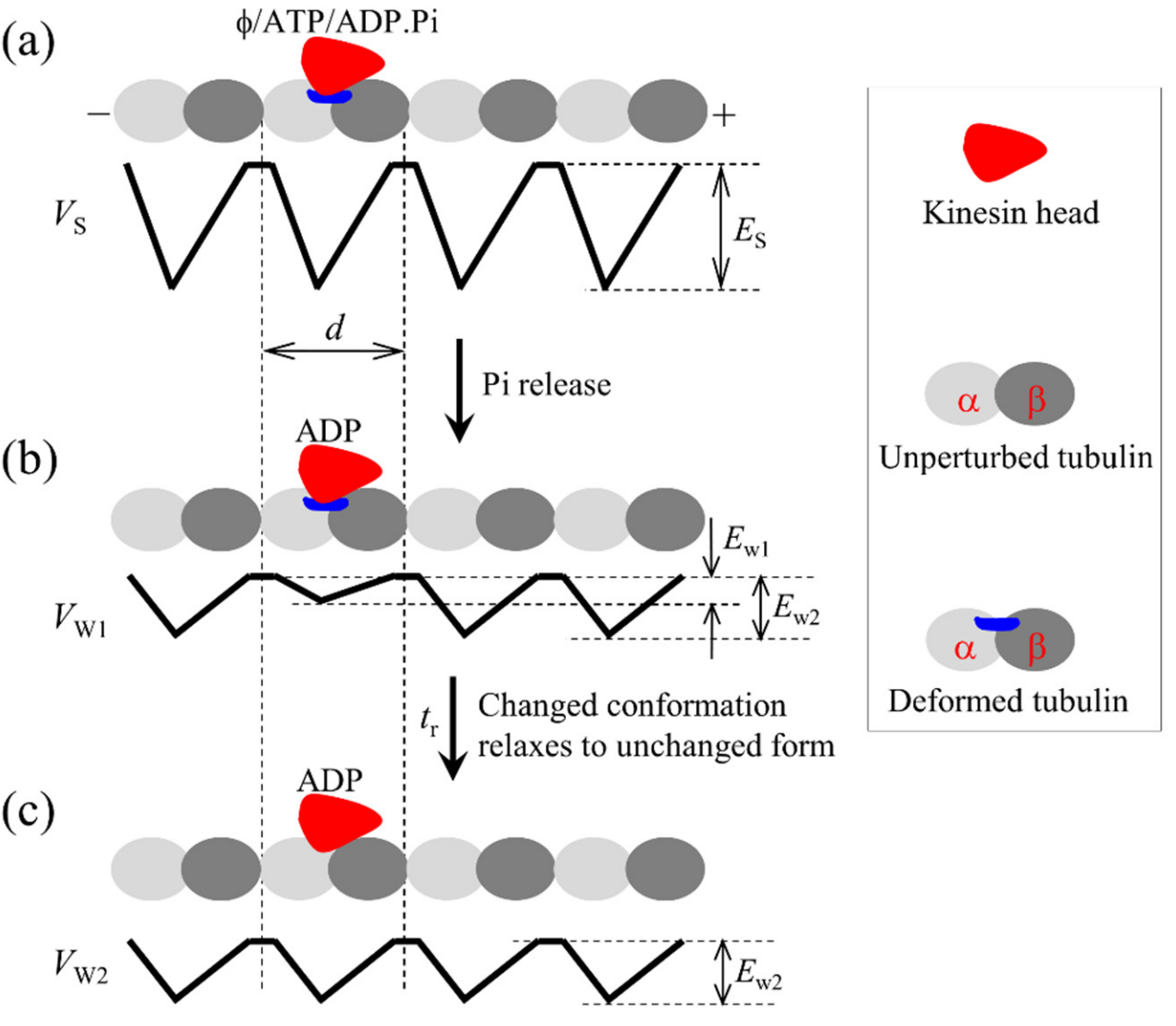

Figure 1. Schematic illustrations of the interaction potential of kinesin head with a MT filament in an ATPase cycle. (a) Potential of the strong interaction of the head in nucleotide-free, ATP and ADP.Pi states with MT. The symbol $\phi$ represents nucleotide free. The strong interaction induces large conformational changes of the local tubulin. (b) Potential of the weak interaction of the head with MT within time $t_{\mathrm{r}}$ after Pi release, when the local tubulin still has the large conformational changes. (c) Potential of the weak interaction of the ADP-head with MT in time $t_{\mathrm{r}}$ after Pi release, when the local tubulin returns to the normally unchanged conformation. Top panels in $(\mathbf{a}-\mathbf{c})$ show schematically the position of the head on the MT filament.

(a)

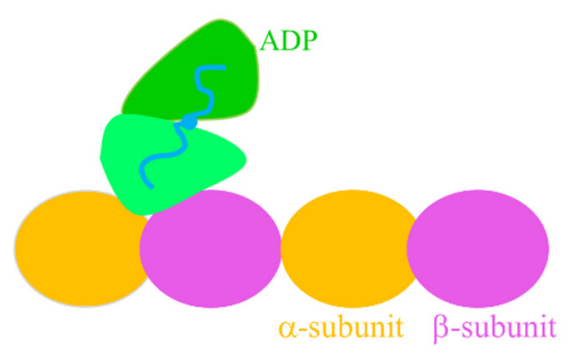

(b)

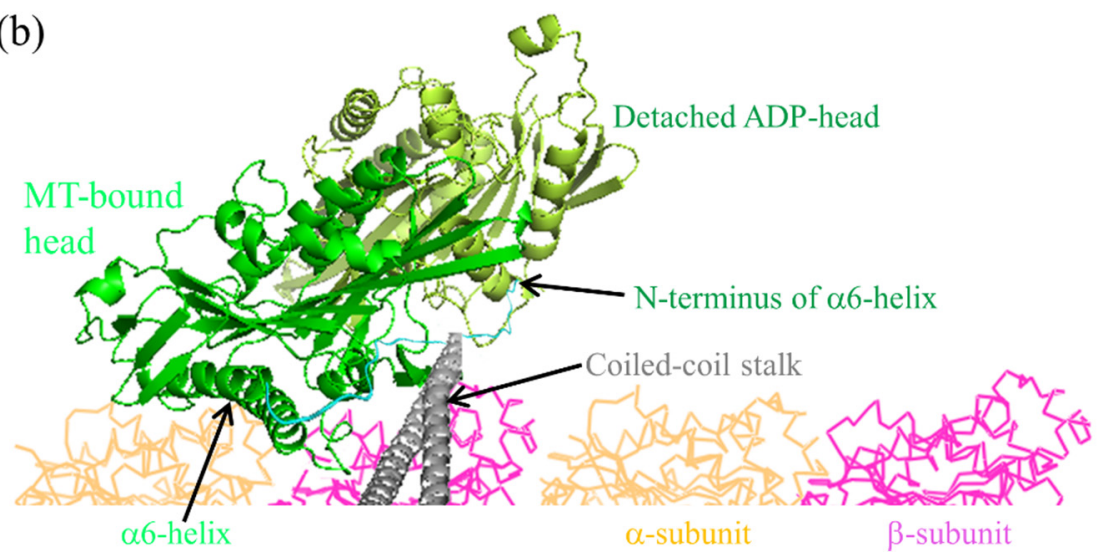

Figure 2. The INT state of kinesin homodimer before the occurrence of the large conformational change of the MTbound head induced by ATP binding, with the two heads having a high affinity. (a) Schematic diagram of the position of the detached ADP-head relative to the MT-bound head. (b) Position and orientation of the detached ADP-head relative to the MT-bound head determined from prior AAMD simulations [40], where the coiled-coil stalk and NLs are drawn schematically. 

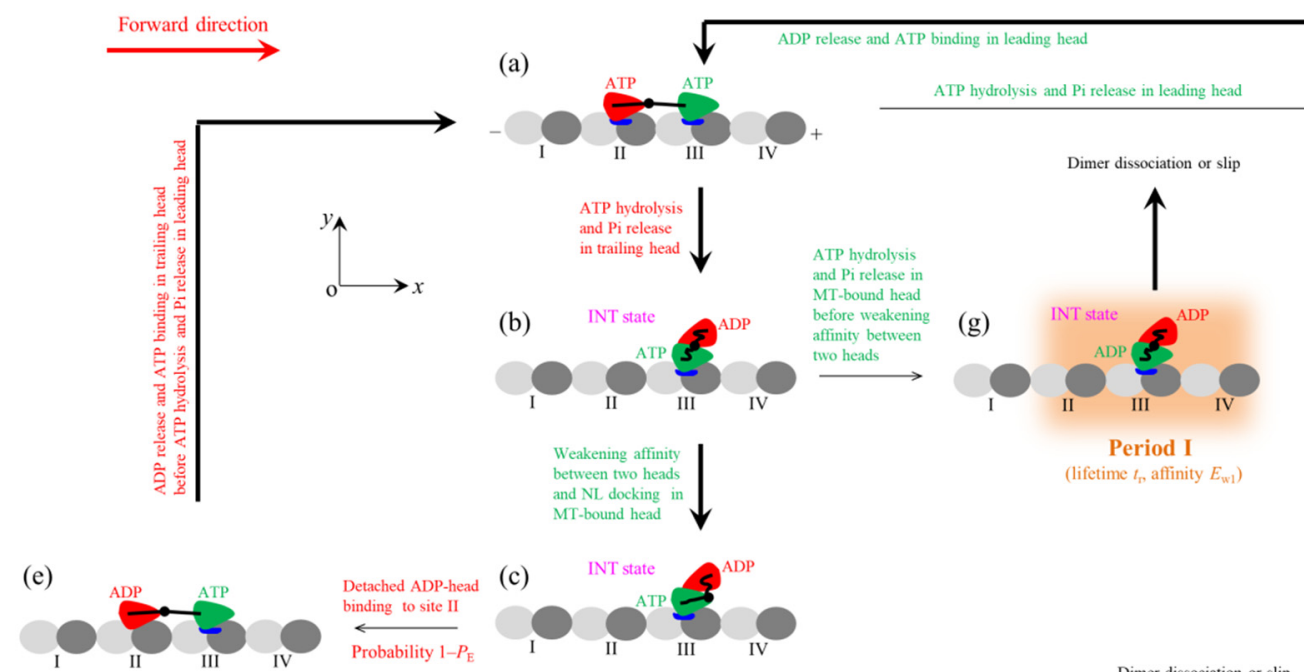
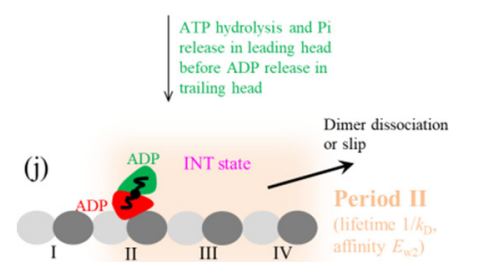

(d)
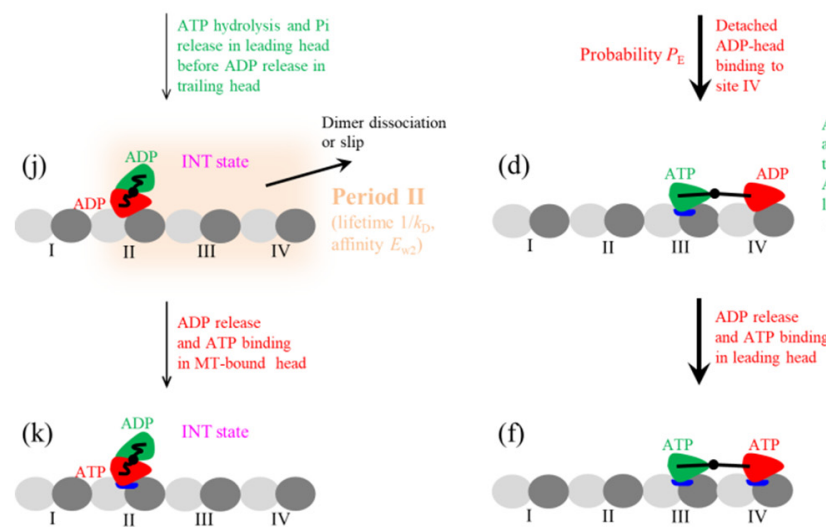

(f)

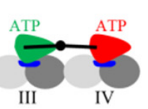

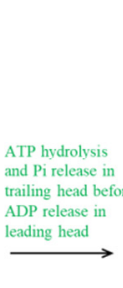
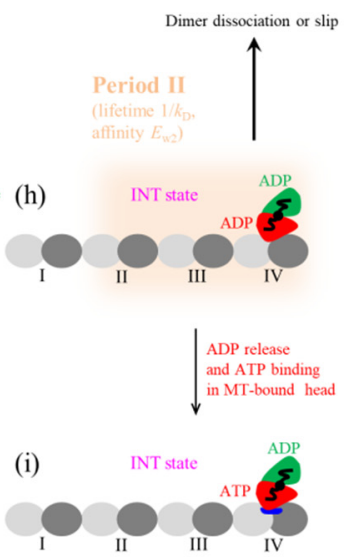

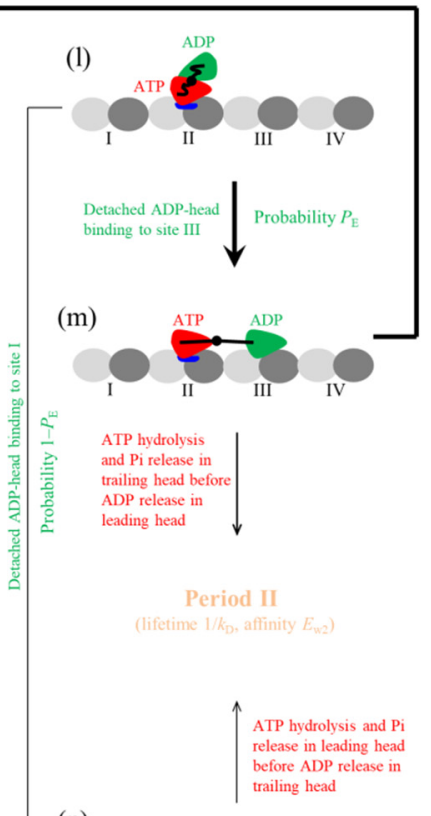

(n)

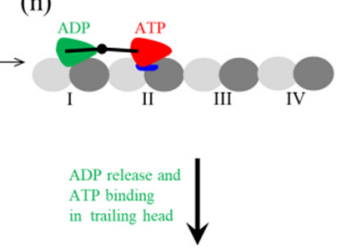

(o)

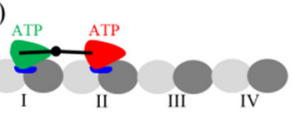

Figure 3. The model for mechanochemical coupling of kinesin homodimer. (a-o) The pathway illustrating kinesin walking, slipping and/or dissociation under saturating ATP concentration (see text for detailed descriptions). Parameter $k_{\mathrm{D}}$ represents ADP-release rate of the MT-bound head. The arrow thickness is approximately proportional to the magnitude of the state transition rate or probability under a low backward load.

We begin with both heads in ATP state, the trailing and leading ones binding strongly to tubulins II and III, respectively, on a MT filament (Figure 3a). First, consider ATP transition to ADP of the trailing head. Within $t_{\mathrm{r}}$ the trailing head detaches readily from tubulin II by overcoming pretty small affinity $E_{\mathrm{w} 1}$ and then diffuses forward. After the detached ADP-head diffusing to the position of the front MT-bound head, the strong interaction between them drives the detached head to move and rotate further to make its $\alpha 6$-helix's N-terminus (to which the NL is connected) be biased in the plus-end position relative to that of the MT-bound head (see Figure 2b) [40]. This state with the two heads having the stable strong interaction is called intermediate (INT) state (Figure 3b, which is the same as Figure 2). Thus, the movement of the rear ADP-head from tubulin II to INT position drives the nano- or micro-sphere attached to the stalk to move forward by a substep of size $d_{1}$. In the INT state, with no NL interference, the closing of NBP and the large conformational change of the MT-bound ATP-head happen rapidly, reducing its binding energy to the partner ADP-head and inducing its NL docking (Figure 3c). Then, the ADP-head can diffuse either forward or backward. The forward diffusion makes the ADP-head bind to the front tubulin IV with affinity $E_{\mathrm{w} 2}$ (Figure 3d). The backward diffusion makes the ADP-head bind to the rear tubulin II with affinity $E_{\mathrm{w} 2}$ by overcoming the energy of the NL docking and the conformational change of the leading ATP-head (Figure 3e) (noting that the affinity of tubulin II for ADP-head has changed to $E_{\mathrm{w} 2}$ in time $t_{\mathrm{r}}$ ). The transition from Figure $3 \mathrm{c}$, d occurs with probability $P_{\mathrm{E}}$ and accordingly the transition from Figure $3 c-e$ occurs with probability $1-P_{\mathrm{E}}$. The binding of the ADP-head to 
MT triggers the release of ADP, followed instantly by ATP binding (Figure 3a,f). Figure $3 f$ is the same as Figure 3a except that the dimer took a forward step.

In Figure $3 b$, before the reduction of the binding energy of the two heads, ATP transition to ADP of the MT-bound head can occur occasionally (Figure 3g). The ADP-head then has pretty small affinity $E_{\mathrm{w} 1}$ for tubulin III for the short time period $t_{\mathrm{r}}$ (termed as Period I), during which the motor can readily dissociate from MT or slip to the adjacent tubulin by overcoming $E_{\mathrm{w} 1}$. After slipping to the adjacent tubulin, the ADP-head has affinity $E_{\mathrm{w} 2}$ for the tubulin. In Figure 3d, before ADP release from the leading head, ATP transition to ADP of the trailing head can also occur. Then, the trailing head detaches readily from tubulin III by overcoming $E_{\mathrm{w} 1}$ and moves to INT position (Figure $3 \mathrm{~h}$ ). In Figure $3 h$, the two heads have the high binding energy and the head has weak affinity $E_{\mathrm{w} 2}$ for MT. The period in this state is termed as Period II, during which the dimer has a large probability to dissociate from MT or slip on MT. If the motor has not dissociated until ADP release from the MT-bound head, after ATP binding (Figure 3i, where no slip was shown) the system becomes the same as Figure $3 \mathrm{~b}$ except that the dimer was moved. In Figure 3e, before ADP release from the trailing head, ATP transition to ADP of the leading head can also occur, with the leading head diffusing to INT position (Figure 3j). During Period II of Figure $3 \mathbf{j}$, the motor has a large probability to dissociate from MT or slip on MT. If the motor has not dissociated until ADP release from the MT-bound head, after ATP binding (Figure 3k, where no slip was shown) the system becomes the same as Figure 3b except that dimer was moved.

Second, consider in Figure 3a ATP transition to ADP of the leading head. The leading head detaches readily from tubulin III by overcoming pretty small affinity $E_{\mathrm{w} 1}$ and then diffuses backward (Figure 31). From Figure 31, the detached ADP-head can either rebind to tubulin III with affinity $E_{\mathrm{w} 2}$ in time $t_{\mathrm{r}}$ (Figure $3 \mathrm{~m}$ ) or bind to tubulin I with affinity $E_{\mathrm{w} 2}$ by overcoming the energy of the NL docking and the conformational change of the ATP-head (Figure $3 \mathrm{n}$ ). Since $t_{\mathrm{r}}$ is very short, the transition from Figure $31-\mathrm{m}$ or to Figure $3 \mathrm{n}$ has approximately the same probability $P_{\mathrm{E}}$ or probability $1-P_{\mathrm{E}}$ as the transition from Figure $3 \mathrm{c}, \mathrm{d}$ or to Figure $3 \mathrm{e}$. The binding of the ADP-head to MT triggers the release of ADP, followed instantly by ATP binding (Figure 3a,o). Figure 30 is the same as Figure $3 a$ except that the motor took a backward step.

In Figure $3 \mathrm{~m}$, before ADP release from the leading head ATP transition to ADP can also occur in the trailing head, with the trailing head diffusing to INT position and the motor being in Period II. In Figure 3n, before ADP release from the trailing head ATP transition to ADP can also occur in the leading head, with the leading head diffusing to INT position and the motor being in Period II. During Period II, the motor has a large probability to dissociate from MT or slip on MT.

\section{Stepping Dynamics}

As shown before [18,24-27,42], with the above model various single molecule data about velocity, run length, forward to backward stepping ratio, dissociation rate, mechanochemical coupling ratio, asymmetric or limping stepping, etc., for both wild-type kinesin-1 homodimers and the corresponding mutant ones with extension of their NLs can be explained quantitatively and consistently. In particular, the puzzling experimental results showing that under no load the wild-type human kinesin-1 consumes about one ATP per mechanical step whereas the mutant one consumes about 3.5 ATPs per mechanical step were explained well $[25,26]$. The puzzling single molecule data showing that the run length of Drosophila kinesin- 1 is very asymmetrical under the forward and backward loads on the stalk, with the run length for a moderate forward load being much smaller than for the backward load of the similar magnitude, were explained well $[18,24,27]$. The puzzling single molecule results showing that the dissociation rate of Drosophila kinesin-1 from MT under the backward load exhibits the characteristic of slip-catch-slip bonds were explained well [18,24,27]. In the following, based on the model we explain the recent experimental results about 
substeps and/or one-head-bound (1HB) and two-heads-bound (2HB) states of kinesin-1 in the stepping cycle.

We firstly present the expression for effective probability $P_{\mathrm{E}}$ (see Figure 3 ) as the function of the load. Due to the flexibility, NL cannot support the compressive force on the two ends and can solely support the stretching force. Consequently, the backward load on the stalk can only act on the NL of the head in the leading position. Hence, during diffusion of the detached head from the rear tubulin to the front MT-bound head, the backward load on the stalk acts mainly on the NL of the leading MT-bound head. The load thus would nearly have no effect on this diffusion. Then, during the further movement and rotation of the detached head to the finally stable INT state, the load on the stalk acts mainly on the NL of the detached head. Due to the strong interaction between the two heads, after the detached head reaching the front MT-bound head the detached head would have nearly $100 \%$ probability to transit to INT state even under a large backward load. Thus, under any load after the trailing head detaching from the rear tubulin it would have a nearly $100 \%$ probability to transit to INT state. From the INT state, the rate for the detached head to diffuse forward and then bind to the front tubulin can be approximately expressed as $k_{\mathrm{F}}=\left(1 / t_{0}\right) \exp \left(-\beta F d^{(+)}\right)$, where $1 / t_{0}$ is the rate under no load, $F$ is the backward load, $d^{(+)}$is the characteristic distance and $\beta^{-1}=k_{\mathrm{B}} T$ is Boltzmann constant times the absolute temperature. From the INT state, the rate for the detached head to diffuse backward and then bind to the rear tubulin can be approximately expressed as $k_{\mathrm{R}}=\left(1 / t_{0}\right) \exp \left(\beta E_{\mathrm{D}}\right)$, where $E_{\mathrm{D}}$ is the free energy change of the NL docking and large conformational change of the head in ATP state. The above expression for $k_{\mathrm{R}}$ is under the following consideration. After the reduction of the binding energy of the two heads, firstly the backward load drives the rapid clockwise rotation of the detached head (see Figure 2) and then the detached head diffuses backward. During the latter diffusion, the load acts mainly on NL of the MT-bound head. $P_{\mathrm{E}}$ can be calculated by $P_{\mathrm{E}}=k_{\mathrm{F}} /\left(k_{\mathrm{F}}+k_{\mathrm{R}}\right)$. Substituting above expressions for $k_{\mathrm{F}}$ and $k_{\mathrm{R}}$ into above expression for $P_{\mathrm{E}}$, we have:

$$
P_{\mathrm{E}}=\frac{\exp \left(\beta E_{\mathrm{D}}\right) \exp \left(-\beta F d^{(+)}\right)}{\exp \left(\beta E_{\mathrm{D}}\right) \exp \left(-\beta F d^{(+)}\right)+1} .
$$

Let $k^{(+)}$stand for the rate of ATP transition to ADP in the head (e.g., the trailing head) with its NL in the forward orientation and $k^{(-)}$stand for the rate of ATP transition to ADP in the head (e.g., the leading head) with its NL not in the forward orientation. As stated before [18,24-27], to be in line with the available experimental results [43-46], $k^{(+)}$and $k^{(-)}$ are independent of the force on the two NLs. The available biochemical results indicated that ADP release is a non-rate-limiting step of the ATPase activity of the kinesin-1 head bound to MT [43]. Moreover, the dwell time in INT state is much smaller than the total dwell time between two steps [23]. Thus, from the pathway of Figure 3 the velocity of the motor can be approximately written as:

$$
v=\left[P_{\mathrm{E}} k^{(+)}-\left(1-P_{\mathrm{E}}\right) k^{(-)}\right] d
$$

where $d=8 \mathrm{~nm}$ is the step size equal to the tubulin repeat period in a MT filament. From Equation (2) it is seen that if $P_{\mathrm{E}} k^{(+)}>\left(1-P_{\mathrm{E}}\right) k^{(-)}$the motor moves forward (toward the plus end) and if $P_{\mathrm{E}} k^{(+)}<\left(1-P_{\mathrm{E}}\right) k^{(-)}$the motor moves backward (toward the minus end). Thus, the moving direction of the dimer is determined by two factors. One factor is the relative magnitude between $k^{(+)}$and $k^{(-)}$, depending on the NL orientation. The other one is $P_{\mathrm{E}}$ that is dictated by energy $E_{\mathrm{D}}$ (see Equation (1)), with NL docking increasing $P_{\mathrm{E}}$.

From Equations (1) and (2) it is noted that to calculate the load dependency of velocity, values of four parameters $k^{(+)}, k^{(-)}, E_{\mathrm{D}}$ and $d^{(+)}$are required. As mentioned above, $k^{(-)}<<k^{(+)}$. Here, we fix $k^{(-)}=1 \mathrm{~s}^{-1}$ (Table 1 ). By adjusting values of the other three parameters, with $k^{(+)}=117 \pm 4 \mathrm{~s}^{-1}, E_{\mathrm{D}}=(1 \pm 0.1) k_{\mathrm{B}} T$ and $d^{(+)}=3.1 \pm 0.2 \mathrm{~nm}$ (Table 1$)$, the single molecule data about the load dependency of velocity for the truncated rat kinesin-1 measured by Sudhakar et al. [23] can be reproduced quantitatively (Figure 4a). Notice that the ATPase rate of the motor, $k^{(+)}+k^{(-)}=118 \mathrm{~s}^{-1}$, is in line with the biochemical results [43,47]. The 
small value of $E_{\mathrm{D}}=1 k_{\mathrm{B}} T$ is consistent with the experimental results indicating that the free energy change of the NL docking is smaller than $1 k_{\mathrm{B}} T$ [48] and the AAMD simulations showing that the energy change of the large conformational change of the head induced by the binding of ATP is only about $1 k_{\mathrm{B}} T$ [49]. In addition, from the structural data determined from the AAMD simulations (Figure $2 b$ ), it is noted that the movement of the detached ADP-head from the rear tubulin to INT position would drive the opticallytrapped nanosphere attached to the stalk to move a distance of about $4 \mathrm{~nm}$, giving the size of the first substep $d_{1} \approx 4 \mathrm{~nm}$. Thus, the second substep also has a size of $d_{2}=d-d_{1} \approx 4 \mathrm{~nm}$. These agree with the single molecule results of Sudhakar et al. [23].

Table 1. Values of parameters for truncated rat kinesin-1 under the experimental condition of Sudhakar et al. [23].

\begin{tabular}{ccccccc}
\hline Parameter & $k^{(+)}$ & $k^{(-)}$ & $E_{\mathrm{D}}$ & $d^{(+)}$ & $k_{\mathrm{NL}}$ & $t_{\mathbf{0}}$ \\
\hline & $\left(\mathbf{s}^{-\mathbf{1}}\right)$ & $\left(\mathbf{s}^{-\mathbf{1}}\right)$ & $\left(k_{\mathrm{B}} \boldsymbol{T}\right)$ & $\mathbf{( n m )}$ & $\mathbf{( \mathbf { s } ^ { - \mathbf { 1 } } )}$ & $(\mathbf{m s})$ \\
\hline value & $117 \pm 4$ & 1 & $1 \pm 0.1$ & $3.1 \pm 0.2$ & 1500 & $0.24 \pm 0.03$ \\
\hline
\end{tabular}
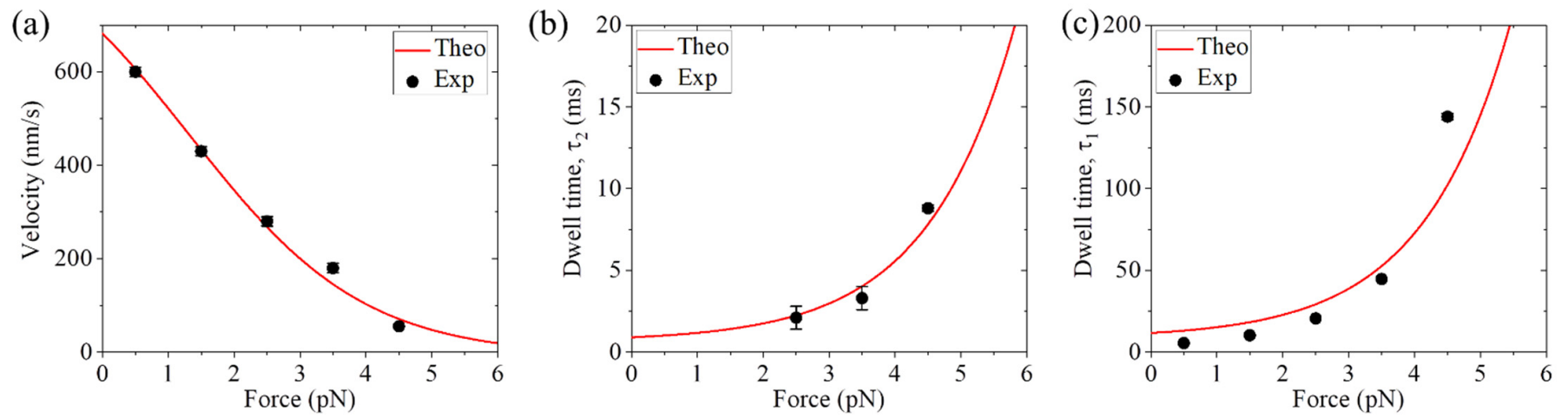

Figure 4. Dynamics of kinesin-1 under the backward load. Lines are theoretical results. Dots are single molecule data measured by Sudhakar et al. [23]. (a) Velocity versus load. (b) Dwell time for forward Substep 2, $\tau_{2}$, versus load. (c) Dwell time for forward Substep 1, $\tau_{1}$, versus load.

Since in the INT state, the large conformational change of the MT-bound ATP-head induces both the reduction of its binding energy to the partner ADP-head and its NL docking, it is expected that the rate of the reduction of the binding energy is nearly the same as that of the NL docking, with both rates being denoted by $k_{\mathrm{NL}}$. Thus, the dwell time for the backward Substep 2 can be written as $\tau_{2}=1 / k_{\mathrm{NL}}+t_{0} \exp \left(\beta E_{\mathrm{D}}\right)$. The dwell time for the forward Substep 2 can be written as:

$$
\tau_{2}=\frac{1}{k_{\mathrm{NL}}}+t_{0} \exp \left(\beta F d^{(+)}\right) .
$$

As conducted in the previous work [24], we take $k_{\mathrm{NL}}=1500 \mathrm{~s}^{-1}$ (see Table 1), which is in line with the biochemical results indicating that the rate constant of NL docking is larger than $800 \mathrm{~s}^{-1}$ [50]. Using Equation (3) and adjusting value of $t_{0}=0.24 \pm 0.03 \mathrm{~ms}$ (Table 1), the single molecule results about the load dependency of dwell time for the forward Substep 2 of the truncated rat kinesin-1 measured by Sudhakar et al. [23] can be reproduced quantitatively (Figure $4 \mathrm{~b}$ ). The value of $t_{0}=240 \mu \mathrm{s}$ is reasonable by considering that the diffusion of the detached head requires overcoming both the large drag force on the 70-nm sphere [23] and the large elastic force arising from NL stretching [25].

In the single molecule assays of Sudhakar et al. [23], due to the rapid and large fluctuations the short dwells of the motor in INT state under the backward load $F<2 \mathrm{pN}$ are unable to distinguish. As shown above, we have $E_{\mathrm{D}}=1 k_{\mathrm{B}} T$ and $d^{(+)}=3.1 \mathrm{~nm}$ (Table 1) for the truncated rat kinesin-1 under the experimental conditions of Sudhakar et al. [23]. Thus, the dwell time for the backward Substep 2 under any backward load is equal to the dwell 
time for the forward Substep 2 under the backward load $F=E_{\mathrm{D}} / d^{(+)}=1.33 \mathrm{pN}$, which is smaller than $2 \mathrm{pN}$. Hence, the short dwells of the motor in INT state for the backward Substep 2 under any backward load are unable to distinguish in the experiments [23]. Accordingly, under any backward load, the measured dwell time $\tau_{1}$ for the forward Substep 1 can be calculated by:

$$
\tau_{1}=\frac{1}{k^{(+)} P_{E}}
$$

Using Equation (4) and parameter values presented in Table 1, the calculated results about the load dependency of dwell time for the forward Substep 1 are also consistent with the single molecule results of Sudhakar et al. [23] (Figure 4c).

In addition, it is seen from the pathway (Figure 3) that only in INT state can the motor has a weak affinity to MT and thus has a large probability to dissociate from MT or slip on MT. This implies that the dissociation or slip of the motor can occur mainly during the dwell period for Substep 2, which is also in line with the single molecule results of Sudhakar et al. [23]. Moreover, from the interaction potential of ADP-head with a MT filament (Figure 1c), it is seen evidently that the motor would slip in discrete steps of size $d=8 \mathrm{~nm}$, also in line with the single molecule results of Sudhakar et al. [23]. The origin that the slip was usually observed in the optical trapping experiments of Sudhakar et al. [23] whereas the dissociation was usually observed in the traditional optical trapping experiments [12,13] can be explained as follows. In the former experiments, a 70-nm sphere was used whereas in the latter experiments a micrometer-sized sphere was used. Thus, the magnitude of the vertical component of the load relative to that of the horizontal component for the former case is much smaller than that for the latter case [42]. Hence, under the large backward load, for the former case the motor would have a larger probability to move along the MT filament (leading to slip) than that to move in the vertical direction (leading to dissociation), whereas for the latter case the motor would have a smaller probability to move along the MT filament (leading to slip) than that to move in the vertical direction (leading to dissociation).

To further verify the model, we provide predicted results about the load dependency of the mechanochemical coupling efficiency, which is defined as the reciprocal of the mean number of ATPs consumed per step (including both forward and backward steps). The number can be written as:

$$
N=\frac{k^{(+)}+k^{(-)}}{P_{\mathrm{E}} k^{(+)}+\left(1-P_{\mathrm{E}}\right) k^{(-)}} .
$$

Using Equation (5) and parameter values presented in Table 1, the predicted results are shown in Figure 5 . It is seen that $N \approx 1.37$ under near zero load, which is close to the prior experimental results [51-53]. The number of ATP molecules hydrolyzed per step rises significantly with the increase in the backward load.

Up to now, we have only focused on saturating ATP. The studies at non-saturating ATP are given in Supplementary Materials. With the pathway of kinesin stepping at nonsaturating ATP (Figure S1), the contradictory single molecule results about the dependency of lifetime of $1 \mathrm{HB}$ state and that of $2 \mathrm{HB}$ state in the stepping cycle on ATP concentration observed by different research groups can be explained quantitatively (Figures S2 and S3). For example, the single molecule results measured by Isojima et al. [54] for cysteine-light human kinesin-1 showed that the lifetime of $1 \mathrm{HB}$ state increases significantly with the decrease of ATP concentration whereas the lifetime of $2 \mathrm{HB}$ state changes insensitively with the change of ATP concentration (Figure S2). By contrast, the single molecule results measured by Mickolajczyk et al. [55] for truncated Drosophila kinesin-1 showed that the lifetime of $2 \mathrm{HB}$ state increases greatly with the decrease of ATP concentration whereas the lifetime of $1 \mathrm{HB}$ state has a small increase with the decrease of ATP concentration (Figure S3). The contradictory results for the ATP-concentration dependency of the lifetime of $1 \mathrm{HB}$ and that of $2 \mathrm{HB}$ are due to different values of the high binding energy between the MTbound nucleotide-free head and the partner ADP-head, which are in turn due to different kinesin-1 species and different buffer conditions used in different assays. Moreover, the 
single molecule results of Sudhakar et al. [23] about the dependencies of velocity, dwell time for the forward Substep 1 and dwell time for the forward Substep 2 on the backward load at low ATP concentration $(10 \mu \mathrm{M})$ for the truncated rat kinesin-1 motor can also be reproduced well (Figure S4).

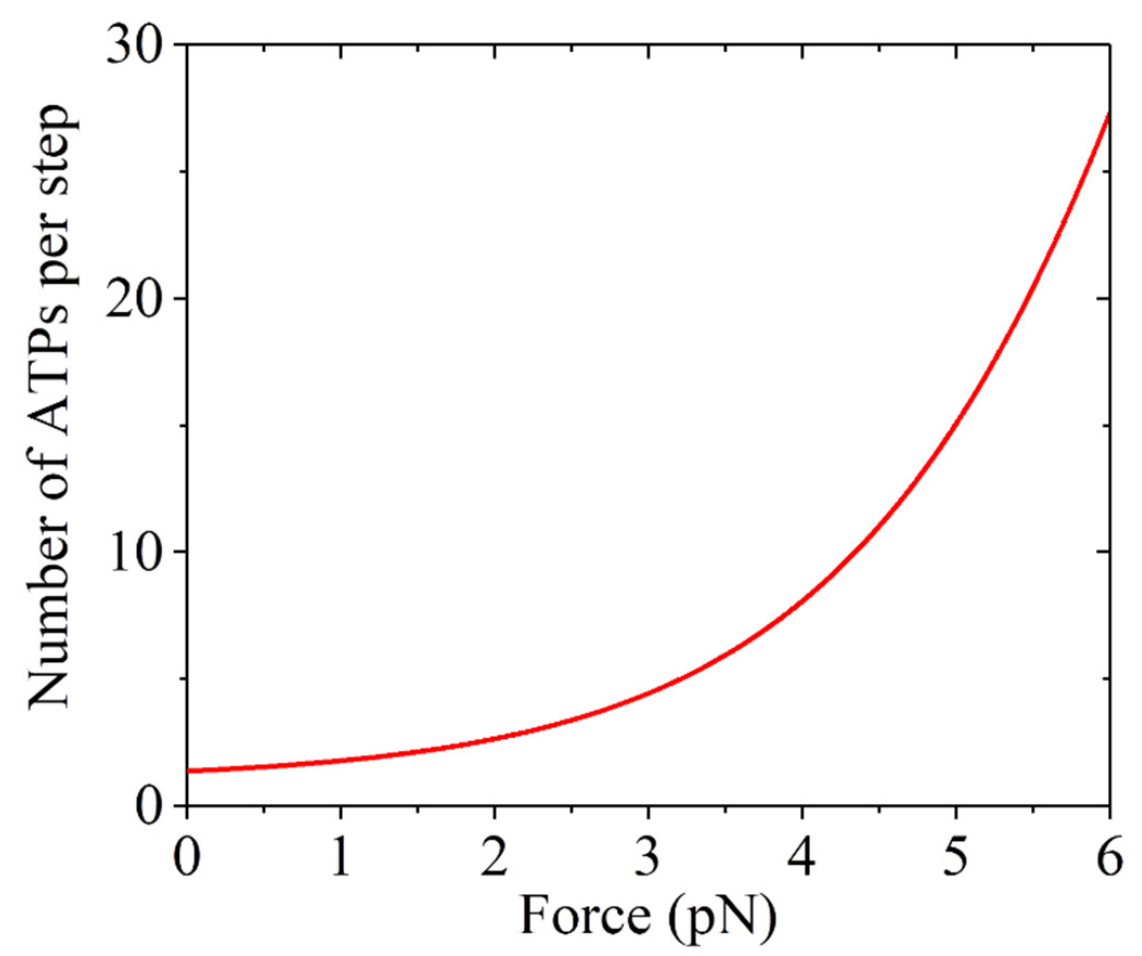

Figure 5. Predicted results for the load dependency of mean number of ATP molecules hydrolyzed per mechanical step.

\section{Concluding Remarks}

The recent single molecule results of Sudhakar et al. about stepping mechanics [23] and the single molecule results about lifetimes of $1 \mathrm{HB}$ and $2 \mathrm{HB}$ states in the stepping cycle $[23,54,55]$ for kinesin- 1 motors are explained well with the model shown schematically in Figure 3. In prior studies, with the model various experimental results for both wild-type kinesin- 1 motors and the mutant ones with extension of their NLs were explained quantitatively and consistently $[18,24-27,42]$. Moreover, with the model, the single molecule results for other families of $\mathrm{N}$-terminal kinesin motors such as kinesin-2, kinesin-3, kinesin-5, kinesin-8, kinesin-12, orphan kinesin PAKRP2, etc., were also explained well and consistently [27-30]. All of these support strongly the model, deepening greatly our understanding of the mechanism of mechanochemical coupling of the N-terminal kinesin motors. To further verify the model it is hoped to test the predicted results (Figure 5) by future experiments.

Supplementary Materials: The following are available online at https: / www.mdpi.com/article / 10.3390 / sym13101799/s1, Figure S1: schematic illustrations of kinesin stepping at non-saturating ATP, Figure S2: dynamics of cysteine-light human kinesin-1 under the experimental condition of Isojima et al. [54], Figure S3: dynamics of truncated Drosophila kinesin-1 under the experimental condition of Mickolajczyk et al. [55], Figure S4: dynamics of truncated rat kinesin-1 under at $10 \mu \mathrm{M}$ ATP under the experimental condition of Sudhakar et al. [23].

Funding: This work was supported by the National Natural Science Foundation of China (Grant No. 11775301).

Institutional Review Board Statement: Not applicable.

Informed Consent Statement: Not applicable. 


\section{Data Availability Statement: Not applicable.}

Conflicts of Interest: The author declares no conflict of interest.

\section{References}

1. Vale, R.D.; Reese, T.S.; Sheetz, M.P. Identification of a novel force-generating protein, kinesin, involved in microtubule-based motility. Cell 1985, 42, 39-50. [CrossRef]

2. Lawrence, C.J.; Dawe, R.K.; Christie, K.R.; Cleveland, D.W.; Dawson, S.C.; Endow, S.A.; Goldstein, L.S.B.; Goodson, H.V.; Hirokawa, N.; Howard, J.; et al. A standardized kinesin nomenclature. J. Cell Biol. 2004, 167, 19-22. [CrossRef]

3. Kozielski, F.; Sack, S.; Marx, A.; Thormahlen, M.; Schonbrunn, E.; Biou, V.; Thompson, A.; Mandelkow, E.-M.; Mandelkow, E. The crystal structure of dimeric kinesin and implications for microtubule-dependent motility. Cell 1997, 91, 985-994. [CrossRef]

4. Yildiz, A.; Tomishige, M.; Vale, R.D.; Selvin, P.R. Kinesin walks hand-over-hand. Science 2004, 303, 676-678. [CrossRef]

5. Asbury, C.L.; Fehr, A.N.; Block, S.M. Kinesin Moves by an Asymmetric Hand-Over-Hand Mechanism. Science 2003, $302,2130$. [CrossRef]

6. Visscher, K.; Schnitzer, M.J.; Block, S.M. Single Kinesin Molecules Studied with a Molecular Force Clamp. Nature 1999, 400, 184-189. [CrossRef]

7. Schnitzer, M.J.; Visscher, K.; Block, S.M. Force production by single kinesin motors. Nat. Cell Biol. 2000, 2, 718-723. [CrossRef]

8. Nishiyama, M.; Higuchi, H.; Yanagida, T. Chemomechanical Coupling of the Forward and Backward Steps of Single Kinesin Molecules. Nat. Cell Biol. 2002, 4, 790-797. [CrossRef] [PubMed]

9. Carter, N.J.; Cross, R.A. Mechanics of the Kinesin Step. Nature 2005, 435, 308-312. [CrossRef]

10. Taniguchi, Y.; Nishiyama, M.; Ishii, Y.; Yanagida, T. Entropy rectifies the Brownian steps of kinesin. Nat. Chem. Biol. 2005, 1, 342-347. [CrossRef] [PubMed]

11. Kawaguchi, K.; Ishiwata, S. Temperature dependence of force, velocity, and processivity of single kinesin molecules. Biochem. Biophys. Res. Commun. 2000, 272, 895-899. [CrossRef]

12. Milic, B.; Andreasson, J.O.L.; Hancock, W.O.; Block, S.M. Kinesin processivity is gated by phosphate release. Proc.Natl. Acad. Sci. USA 2014, 111, 14136-14140. [CrossRef] [PubMed]

13. Andreasson, J.O.L.; Milic, B.; Chen, G.-Y.; Guydosh, N.R.; Hancock, W.O.; Block, S.M. Examining Kinesin Processivity Within a General Gating Framework. eLife 2015, 4, e07403. [CrossRef] [PubMed]

14. Fisher, M.E.; Kolomeisky, A.B. Simple mechanochemistry describes the dynamics of kinesin molecules. Proc. Natl. Acad. Sci. USA 2001, 98, 7748-7753. [CrossRef] [PubMed]

15. Liepelt, S.; Lipowsky, R. Kinesin's network of chemomechanical motor cycles. Phys. Rev. Lett. 2007, 98, 258102. [CrossRef]

16. Hyeon, C.; Onuchic, J.N. Internal strain regulates the nucleotide binding site of the kinesin leading head. Proc.Natl. Acad. Sci. USA 2007, 104, 2175-2180. [CrossRef]

17. Sumi, T. Design principles governing chemomechanical coupling of kinesin. Sci. Rep. 2017, 7, 1163. [CrossRef]

18. Xie, P. Insight into the chemomechanical coupling mechanism of kinesin molecular motors. Commun. Theor. Phys. 2021, 73, 057601. [CrossRef]

19. Hyeon, C.; Klumpp, S.; Onuchic, J.N. Kinesin's backsteps under mechanical load. Phys. Chem. Chem. Phys. 2009, 11, 4899-4910. [CrossRef]

20. Wang, Q.; Diehl, M.R.; Jana, B.; Cheung, M.S.; Kolomeisky, A.B.; Onuchic, J.N. Molecular origin of the weak susceptibility of kinesin velocity to loads and its relation to the collective behavior of kinesins. Proc. Natl. Acad. Sci. USA 2017, 114, E8611-E8617. [CrossRef]

21. Sasaki, K.; Kaya, M.; Higuchi, H. A unified walking model for dimeric motor proteins. Biophys. J. 2018, 115, 1-12. [CrossRef]

22. Mugnai, M.L.; Hyeon, C.; Hinczewski, M.; Thirumalai, D. Theoretical perspectives on biological machines. Rev. Mod. Phys. 2020, 92, 025001. [CrossRef]

23. Sudhakar, S.; Kazem, M.; Tobias, A.; Jachowski, J.; Bugiel, M.; Jannasch, A.; Schäffer, E. Germanium nanospheres for ultraresolution picotensiometry of kinesin motors. Science 2021, 71, eabd9944.

24. Xie, P. Theoretical analysis of dynamics of kinesin molecular motors. ACS Omega 2020, 5, 5721-5730. [CrossRef]

25. Guo, S.-K.; Wang, P.-Y.; Xie, P. A model of processive movement of dimeric kinesin. J. Theor. Biol. 2017, 414, 62-75. [CrossRef]

26. Xie, P.; Guo, S.-K.; Chen, H. A generalized kinetic model for coupling between stepping and ATP hydrolysis of kinesin molecular motors. Int. J. Mol. Sci. 2019, 20, 4911. [CrossRef]

27. Xie, P. Dynamics of kinesin motor proteins under longitudinal and sideways loads. J.Theor. Biol. 2021, 530, 110879. [CrossRef]

28. Guo, S.-K.; Shi, X.-X.; Wang, P.-Y.; Xie, P. Run length distribution of dimerized kinesin-3 molecular motors: Comparison with dimeric kinesin-1. Sci. Rep. 2019, 9, 16973. [CrossRef]

29. Guo, S.-K.; Xie, P. A common chemomechanical coupling model for orphan and conventional kinesin molecular motors. Biophys. Chem. 2020, 264, 106427. [CrossRef] [PubMed]

30. Xie, P. A model of processive walking and slipping of kinesin-8 molecular motors. Sci. Rep. 2021, 11, 8081. [CrossRef] [PubMed]

31. Crevel, I.M.T.C.; Lockhart, A.; Cross, R.A. Weak and Strong States of Kinesin and ncd. J. Mol. Biol. 1996, 257, 66-76. [CrossRef]

32. Cross, R.A. Mechanochemistry of the kinesin-1 ATPase. Biopolymers 2016, 105, 476-482. [CrossRef]

33. Sosa, H.; Peterman, E.J.G.; Moerner, W.E.; Goldstein, L.S.B. ADP-induced rocking of the kinesin motor domain revealed by single-molecule fluorescence polarization microscopy. Nat. Struc. Biol. 2001, 8, 540-544. [CrossRef] 
34. Morikawa, M.; Yajima, H.; Nitta, R.; Inoue, S.; Ogura, T.; Sato, C.; Hirokawa, N. X-ray and Cryo-EM structures reveal mutual conformational changes of kinesin and GTP-state microtubules upon binding. EMBO J. 2015, 34, 1270-1286. [CrossRef]

35. Shi, X.-X.; Wang, P.-Y.; Chen, H.; Xie, P. Studies of conformational changes of tubulin induced by interaction with kinesin using atomistic molecular dynamics simulations. Int. J. Mol. Sci. 2021, 22, 6709. [CrossRef]

36. Shang, Z.; Zhou, K.; Xu, C.; Csencsits, R.; Cochran, J.C.; Sindelar, C.V. High-resolution structures of kinesin on microtubules provide a basis for nucleotide-gated force-generation. eLife 2014, 3, e04686. [CrossRef]

37. Sindelar, C.V.; Downing, K.H. An atomic-level mechanism for activation of the kinesin molecular motors. Proc. Natl. Acad. Sci. USA 2010, 107, 4111-4116. [CrossRef] [PubMed]

38. Rice, S.; Lin, A.W.; Safer, D.; Hart, C.L.; Naber, N.; Carragher, B.O.; Cain, S.M.; Pechatnikova, E.; Wilson-Kubalek, E.M.; Whittaker M.; et al. A structural change in the kinesin motor protein that drives motility. Nature 1999, 402, 778-784. [CrossRef] [PubMed]

39. Asenjo, A.B.; Weinberg, Y.; Sosa, H. Nucleotide binding and hydrolysis induces a disorder-order transition in the kinesin neck-linker region. Nat. Struc. Mol. Biol. 2006, 13, 648-654. [CrossRef] [PubMed]

40. Shi, X.-X.; Guo, S.-K.; Wang, P.-Y.; Chen, H.; Xie, P. All-atom molecular dynamics simulations reveal how kinesin transits from one-head-bound to two-heads-bound state. Proteins 2020, 88, 545-557. [CrossRef] [PubMed]

41. Cao, L.; Wang, W.; Jiang, Q.; Wang, C.; Knossow, M.; Gigant, B. The structure of apo-kinesin bound to tubulin links the nucleotide cycle to movement. Nat. Commun. 2014, 5, 5364. [CrossRef]

42. Guo, S.-K.; Wang, P.-Y.; Xie, P. Dynamics of dimeric kinesins: Limping, effect of longitudinal force, effects of neck linker extension and mutation, and comparison between kinesin-1 and kinesin-2. Int. J. Biol. Macromol. 2017, 105, 1126-1137. [CrossRef]

43. Moyer, M.L.; Gilbert, S.P.; Johnson, K.A. Pathway of ATP hydrolysis by monomeric and dimeric kinesin. Biochemistry 1998, 37, 800-813. [CrossRef]

44. Okada, Y.; Hirokawa, N. A processive single-headed motor: Kinesin superfamily protein KIF1A. Science 1999, 283, 1152-1157. [CrossRef]

45. Rosenfeld, S.S.; Fordyce, P.M.; Jefferson, G.M.; King, P.H.; Block, S.M. Stepping and stretching: How kinesin uses internal strain to walk processively. J. Biol. Chem. 2003, 278, 18550-18556. [CrossRef]

46. Yildiz, A.; Tomishige, M.; Gennerich, A.; Vale, R.D. Intramolecular Strain Coordinates Kinesin Stepping Behavior along Microtubules. Cell 2008, 134, 1030-1041. [CrossRef]

47. Gilbert, S.P.; Johnson, K.A. Pre-Steady-State Kinetics of the Microtubule-Kinesin ATPase. Biochemistry 1994, 33, 1951-1960. [CrossRef]

48. Rice, S.; Cui, Y.; Sindelar, C.; Naber, N.; Matuska, M.; Vale, R.; Cooke, R. Thermodynamic properties of the kinesin neck region docking to the catalytic core. Biophys. J. 2003, 84, 1844-1854. [CrossRef]

49. Hwang, W.; Lang, M.J.; Karplus, M. Kinesin motility is driven by subdomain dynamics. eLife 2017, 6, e28948. [CrossRef] [PubMed]

50. Rosenfeld, S.S.; Jefferson, G.M.; King, P.H. ATP reorients the neck linker of kinesin in two sequential steps. J. Biol. Chem. 2001, 276, 40167-40174. [CrossRef] [PubMed]

51. Hua, W.; Young, E.C.; Fleming, M.L.; Gelles, J. Coupling of kinesin steps to ATP hydrolysis. Nature 1997, 388, 390-393. [CrossRef] [PubMed]

52. Coy, D.L.; Wagenbach, M.; Howard, J. Kinesin Takes One 8-nm Step for Each ATP That It Hydrolyzes. J. Biol. Chem. 1999, 274, 3667-3671. [CrossRef] [PubMed]

53. Schnitzer, M.J.; Block, S.M. Kinesin hydrolyses one ATP per 8-nm step. Nature 1997, 388, 386-390. [CrossRef]

54. Isojima, H.; Iino, R.; Niitani, Y.; Noji, H.; Tomishige, M. Direct observation of intermediate states during the stepping motion of kinesin-1. Nat. Chem. Biol. 2016, 12, 290-297. [CrossRef]

55. Mickolajczyk, K.J.; Deffenbaugh, N.C.; Arroyo, J.O.; Andrecka, J.; Kukura, P.; Hancock, W.O. Kinetics of nucleotide-dependent structural transitions in the kinesin-1 hydrolysis cycle. Proc. Natl. Acad. Sci. USA 2015, 112, E7186-E7193. [CrossRef] 